\title{
Electrical Impedance Tomography might be a Practical Tool to Pro- vide Information about COVID-19 Pneumonia Progression
}

Rongqing Chen, Institute of Technical Medicine, Furtwangen University, Jakob-Kienzle-Str. 17, VS-Schwenningen, Germany. e-mail: chr@hs-furtwangen.de

András Lovas, Kiskunhalas Semmelweis Hospital, Department of Anaesthesiology and Intensive Therapy, H-6400, Dr. Monszpart L. u. 1, Hungary. e-mail: anlovas@gmail.com

Balázs Benyó: Budapest University of Technology and Economics, Department of Control Engineering and Information Technology, Ñ117 Budapest, Magyar tudósok krt. 2, Hungary. e-mail: bbenyo@iit.bme.hu

Knut Moeller: Institute of Technical Medicine, Furtwangen University, Jakob-Kienzle-Str. 17, VS-Schwenningen, Germany. e-mail: moe@fs-furtwangen.de

\section{Introduction}

COVID-19 induced acute respiratory distress syndrome (ARDS) could have two different phenotypes, namely L-type and H-type. Different phenotypes were reported to have different responses and outcomes to the typical ARDS positive end-expiration pressure (PEEP) treatment. The identification of the different phenotypes in terms of the PEEP recruitment difference can help to improve the patient outcome.

\section{Methods}

This contribution was conducted with a retrospective patient dataset. The patient was diagnosed with COVID-19 pneumonia and admitted to intensive care unit. PEEP trial was applied on the patient with electrical impedance tomography (EIT) monitoring for seven days. Conductivity distribution difference images between the PEEP level 25 and $10 \mathrm{cmH}_{2} \mathrm{O}$ were obtained from tidal variation images on day 1, day 3 and day 7, respectively. The conductivity distribution difference images were used to detect the recruitment areas during the PEEP trial.

\section{Results}

A clear course developing trend in terms of decreasing recruitment area can be observed in conductivity distribution difference images, which is complied with the medical record stating a deteriorating condition of the patient. This analysis indicate that the patient is a L-type with low recruitability. If this is the case, the traditional recruitment maneuvers used in common ARDS patients might not provide the expected outcome, on the contrary might introduce an increased risk of structural damage of the lung due to the high PEEP.

\section{Conclusion}

The course of the COVID-19 pneumonia is still poorly understood and has shown to develop very fast. In this contribution, a rather clear development of a L-type patient can be observed from the long-term EIT monitoring. EIT might develop into a useful and practical tool to assist with the classification of the different phenotypes of the COVID-19 patients in addition to the CT, and might provide additional information about progression of the disease and the evaluation of its treatment strategies. 


\title{
eVision: Epidemic Forecasting on COVID-19
}

Navid Shaghaghi, Andres Calle, George Kouretas, Jaidev Mirchandani, and Michael Castillo EPIC and Bioinnovation and Design Labs, Santa Clara University, Santa Clara, California, USA

\{nshaghaghi, acalle, gkouretas, jmirchandani, mvcastillo\}@scu.edu

\begin{abstract}
Vaccination is the primary strategy to prevent COVID-19 illness and hospitalization. However, supplies are scarce and due to the regional mutations of the virus, new vaccines or booster shots will need to be administered every so often. Hence, the prediction of the rate of growth in reported COVID-19 cases is paramount to ensuring the ample supply of vaccines as well as for local/state/federal government measures to ensure the availability of hospital beds, supplies, and staff.

eVision is an epidemic forecaster aimed at combining AI - in the form of a Long Short-Term Memory (LSTM) Recursive Neural Network (RNN) - and search engine statistics, in order to make accurate predictions about the weekly number of cases for highly communicable diseases. Starting on replicating an older Google model and then improving upon it, predictions are accurately made as far as seven weeks into the future with an accuracy rate of $\% 91$ for seasonal influenza.

While many different kinds of forecasting models have been created to track the COVID-19 pandemic, they have missed the insight discovered by eVision on influenza: by simply providing the AI model with the relative popularity of carefully selected key phrases searched via Google along with the number of positive cases reported from the CDC and/or WHO the model can make highly accurate predictions about the trend of the outbreak by learning the relationship between the two trends. eVision is thus provided with a weekly count of the number of COVID-19 cases in a region along with statistics surrounding common search phrases such as "loss of smell" and "loss of taste" that have been searched via Google in that region since the start of the pandemic. It has, for instance, been able to achieve an accuracy of $\% 89$ for predicting the trend of the COVID-19 outbreak in the United States.
\end{abstract}




\section{Safety and Automation Concepts for Artificial Implantable Lungs - SmartLungControl}

Michael Lamberti, Chair of Computer Science 11 - Embedded Software, RWTH Aachen University, Aachen, Germany, lamberti@embedded.rwth-aachen.de

Rüdger Kopp, Clinic for Operative Intensive Medicine and Intermediate Care, Uniklinik RWTH Aachen, Aachen, Germany, rkopp@ukaachen.de

Cavan Lübke, Clinic for Operative Intensive Medicine and Intermediate Care, Uniklinik RWTH Aachen, Aachen, Germany, caluebke@ukaachen.de

Klaus Steffen Leonhardt, Chair for Medical Information Technology, RWTH Aachen University, Aachen, Germany, leonhardt@hia.rwth-aachen.de

Marian Walter, Chair for Medical Information Technology, RWTH Aachen University, Aachen, Germany, walter@hia.rwth-aachen.de

André Stollenwerk, Chair of Computer Science 11 - Embedded Software, RWTH Aachen University, Aachen, Germany, stollenwerk@embedded.rwth-aachen.de

\section{Introduction}

An artificial implantable lung - the long-term goal of the SPP 2014 - will enable patients suffering from severe lung disease to participate in normal life. Changing physiological conditions, such as walking, climbing stairs or resting, imply dynamically changing physiological demand. This requires the implantable lung to operate safely on a large scale of working points. While today's lung support therapies are performed in an intensive care unit under direct supervision of clinical experts, good knowledge of the current condition of the implanted lung is mandatory to ensure a safe and autonomous long-term operation in an ambulatory setting. As a consequence, this project will address the new challenges of autonomous operation.

\section{Methods}

We want to investigate a concept for a demand-adapted controller and safety monitoring of a long-term artificial lung. Based on existing patient and animal trial data, a requirements analysis will be carried out. Information about common complications in lung support therapy will be gathered by reaching out with a structured questionnaire to experts using the Delphi method. In the second step, a controller design and safety concept for the autonomous artificial lung, will be researched and evaluated. New sensor concepts for the patient's physiological demand and the performance of the artificial lung will be developed. Finally, the prototype will be validated and tested in-silico, in-vitro using the hardware-inthe-loop test bench developed by another project within the framework of the SPP2014, and in-vivo in animal experiments. Various basic conditions and changes induced by mobilization will be investigated, as well as defined critical operating states.

\section{Conclusion}

Improving on our existing automation concepts for the extracorporeal lung support, we will focus on new requirements due to the mobilization of patients. As a result of our research, we will provide new concepts for the safe, demand-adapted, and individualized operation of a long-term-artificial lung outside of an intensive care unit. 